\title{
Avaliação perceptiva e instrumental da função velofaríngea na fissura de palato submucosa assintomática ${ }^{* * * * *}$
}

\author{
Perceptual and instrumental assessment of velopharyngeal function \\ in asymptomatic submucous cleft palate
}

\author{
Haline Coracine Miguel* \\ Kátia Flores Genaro** \\ Inge Elly Kiemle Trindade***
}

*Fonoaudióloga. Mestre em Distúrbios da Comunicação Humana pelo Hospital de Reabilitação de Anomalias Craniofaciais (HRCA) da Universidade de São Paulo. Endereço para correspondência: Rua Sílvio Marchione, 3-20 Bauru - SP CEP 17012-900

(halinemiguel@hotmail.com).

**Fonoaudióloga. Professora Associada do Departamento de Fonoaudiologia da Faculdade de Odontologia de Bauru.

Pesquisadora do Laboratório de Fisiologia do HRCA da Universidade de São Paulo.

****Biomédica. Professora Titular do Departamento de Ciências Biológicas da Faculdade de Odontologia de Bauru da Universidade de São Paulo.

Pesquisadora do Laboratório de Fisiologia do HRCA da Universidade de São Paulo.

****Trabalho Realizado no Laboratório de Fisiologia do HRCA da Universidade de São Paulo, Bauru São Paulo.

Artigo de Pesquisa

Artigo Submetido a Avaliação por Pares

Conflito de Interesse: não

Recebido em 21.12.2004.

Revisado em 01.11.2005; 29.09.2006; 23.02.2007.

Aceito para Publicação em 23.02.2007.

\section{Abstract}

Background: submucous cleft palate (SMCP) may or may not be associated to symptoms of velopharyngeal dysfunction (VPD). Aim: to verify whether patients with SMCP, who were considered as asymptomatic on a perceptual speech evaluation, present absence of hypernasality and adequate velopharyngeal closure on an instrumental assessment. Method: 22 patients with SMCP and with no VPD symptoms, of both genders, aged 6 to 46 years, underwent speech acoustic assessment (nasometry) - to determine nasalance scores - and speech aerodynamic assessment (pressure-flow technique) - to verify velopharyngeal closure. The complete agreement between the findings on the perceptual and instrumental assessments was the tested null hypothesis. Results: the pressure-flow technique confirmed the perceptual findings, that is, all patients were diagnosed as having adequate velopharyngeal function on both types of assessment. Nasometry results confirmed the perceptual findings in 15 out of the 20 analyzed patients (75\% of the cases). The remaining 5 patients $(25 \%)$ presented nasalance scores that suggest hypernasality (nasometry). This symptom was not identified by the perceptual evaluation, yielding to the rejection of the null hypothesis. Conclusion: results indicate the importance of the combined use of perceptual and instrumental assessments for the diagnosis of VPD in SMCP cases. The follow-up of patients diagnosed as asymptomatic by a perceptual speech evaluation and presenting evidences of VPD on instrumental assessment (e.g. nasometry) is recommended, particularly when dealing with children who are more prone to develop symptoms with aging.

Key Words: Cleft Palate; Velopharyngeal Insufficiency; Rhinomanometry.

\section{Resumo}

Tema: a fissura de palato submucosa (FPSM) pode estar associada, ou não, a sintomas de disfunção velofaríngea (DVF). Objetivo: o presente estudo teve por propósito verificar se pacientes com FPSM diagnosticados como assintomáticos em uma avaliação perceptiva da fala apresentam ausência de hipernasalidade e fechamento velofaríngeo adequado em exame instrumental. Método: vinte pacientes com FPSM e sem sintomas de DVF, de ambos os gêneros, com idade entre 6 e 46 anos, foram submetidos à avaliação acústica da fala (nasometria), para a determinação da nasalância, o correlato acústico da nasalidade, e, à avaliação aerodinâmica da fala (técnica fluxo-pressão), para a determinação do fechamento velofaríngeo. A total concordância entre os resultados aferidos na avaliação perceptiva e nas avaliações instrumentais foi a hipótese de nulidade testada. Resultados: a avaliação aerodinâmica confirmou integralmente as observações da avaliação perceptiva, ou seja, todos os 20 pacientes foram diagnosticados como tendo fechamento velofaríngeo adequado em ambas as modalidades de avaliação. Os resultados da nasometria, por sua vez, concordaram com os da avaliação perceptiva em apenas 15 dos 20 pacientes analisados $(75 \%$ dos casos). Os 5 pacientes restantes $(25 \%)$ apresentaram escores de nasalância sugestivos de hipernasalidade na nasometria, não constatada na avaliação perceptiva, levando, neste caso, à rejeição da hipótese de nulidade. Conclusão: os resultados mostram a importância do uso combinado de avaliação perceptiva e instrumental para o diagnóstico da DVF em casos de FPSM. Com base nos achados recomenda-se o acompanhamento periódico dos casos considerados assintomáticos em avaliação perceptiva da fala e que apresentem evidências de DVF em uma avaliação instrumental, como a nasometria, particularmente em se tratando de crianças, mais sujeitas ao desenvolvimento de sintomas com o avanço da idade.

Palavras-Chave: Fissura Palatina; Disfunção Velofaríngea; Rinomanometria.

Referenciar este material como:

1 MIGUEL. H. C.; GENARO. K. F; TRINDADE. I. E. K.Avaliação perceptiva e instrumental da função velofaríngea na fissura de palato

$\sum 3$ submucosa assintomática. Pró-Fono Revista de Atualização Científica, Barueri (SP), v. 19, n. 1, p. 105-112, jan.-abr. 2007. 


\section{Introdução}

A fissura de palato submucosa (FPSM) é uma malformação congênita que se caracteriza pela falta de fusão das estruturas ósseas e/ou musculares na linha média do palato, estando intacta a mucosa oral (Genaro et al., 2004). É neste último aspecto que a FPSM se diferencia das fissuras de palato, as quais envolvem também o plano mucoso.

O diagnóstico da FPSM é usualmente feito com base na observação, por inspeção visual e tátil da cavidade oral, dos seguintes sinais anatômicos: úvula bífida, diástase muscular e entalhe ósseo na borda posterior do palato duro (Sommerlad et al., 2004). Esses sinais podem aparecer isoladamente ou de forma combinada. Em estudo desenvolvido por Oliveira (2002) em 50 crianças com FPSM, entre 20 a 40 meses de idade, selecionadas pelo período de um ano por ordem de agendamento entre os pacientes em atendimento no Hospital de Reabilitação de Anomalias Craniofaciais (HRAC/ USP), verificou-se que $50 \%$ dos casos apresentavam a tríade clássica, $30 \%$ apresentavam dois dos sinais clínicos e os outros $20 \%$, apenas a diástase muscular.

A FPSM, com relativa freqüência, está associada a distúrbios de fala-hipernasalidade, escape aéreo nasal e articulações compensatórias - decorrentes de disfunção velofaríngea (DVF), provocada pelo defeito anatômico palatino (Park et al., 2000; Kummer, 2001; Senders, 2001; Ysunza et al., 2001; Sommerlad et al., 2002; Yamashita et al., 2002; Johns et al., 2003; Bataiola e Yamashita, 2005). Em geral, são estes sintomas que levam o paciente à centros de tratamento permitindo a identificação deste tipo de fissura como fatores etiológicos e o posterior tratamento.

Por razões ainda não esclarecidas, a FPSM pode não levar a sintomas de DVF. McWilliams (1991) verificou, em uma análise retrospectiva da fala de 130 pacientes com FPSM, assistidos no Cleft Palate-Craniofacial Center da Universidade de Pittsburgh-EUA, que $44 \%$ deles mostraram-se assintomáticos até a idade adulta, não necessitando de qualquer tipo de intervenção cirúrgica. Do total de casos avaliados no HRAC/ USP por Oliveira (2002), 80\% foram categorizados como assintomáticos. A diferença na proporção de assintomáticos nos dois estudos provavelmente decorre dos critérios de diagnóstico utilizados. Independente de sua real prevalência, fato é que parcela considerável de pacientes com FPSM apresenta-se assintomáticos. $\mathrm{Na}$ tentativa de encontrar justificativa para a ocorrência ou não de
DVF, Park (2002) compararam a morfologia craniofacial de crianças com FPSM sintomáticas $(\mathrm{n}=22)$ e assintomáticas $(\mathrm{n}=24)$, por meio de cefalometria. Observaram diferenças para algumas variáveis aferidas; entretanto, os dados cefalométricos não se mostraram úteis como preditores da função velofaríngea nos pacientes analisados.

No HRAC/USP, uma vez diagnosticada clinicamente a FPSM por uma equipe multidisciplinar, a fala é avaliada por meio de análise perceptiva (Genaro et al., 2004). Se confirmada a ausência de sintomas de DVF, o paciente é esclarecido quanto aos sintomas que possam vir a se desenvolver e orientado a procurar o serviço no caso de sua ocorrência. Isto feito, o paciente é dispensado do tratamento convencional dado aos portadores de fissura. Considerando a natureza subjetiva da avaliação perceptiva da função velofaríngea, a questão que se colocou foi a seguinte: pacientes considerados assintomáticos não estariam sendo indevidamente dispensados de atendimento? Essa argumentação encontra amparo no fato de que o julgamento perceptivo da função velofaríngea é influenciado por fatores como o padrão articulatório do paciente, tonalidade e intensidade da voz, contexto fonético, experiência prévia e expectativas do julgador (Kummer 2001; Dalston 2004).

Assim sendo, o objetivo do presente estudo foi verificar se pacientes com FPSM considerados assintomáticos numa avaliação perceptiva da fala tem efetivamente função velofaríngea adequada, quando esta é aferida por métodos mais objetivos de diagnóstico. Para responder à questão lançouse mão da nasometria e da técnica fluxo-pressão.

A nasometria permite aferir, indiretamente, a adequação do mecanismo velofaríngeo pela medida da nasalância, uma grandeza física que corresponde à quantidade relativa de energia acústica emitida através da cavidade nasal durante a fala; a nasalância é o correlato acústico da nasalidade. $\mathrm{O}$ método parte do pressuposto que um aumento da nasalância na produção de amostras de fala contendo exclusivamente sons orais é sugestivo de hipernasalidade, um dos sinais da DVF. A técnica fluxo-pressão, por sua vez, permite aferir a adequação do mecanismo velofaríngeo pela medida simultânea do fluxo nasal e das pressões oral e nasal geradas na fala. É o único método capaz de quantificar as dimensões do orifício velofaríngeo na fala, mais precisamente, 
a sua área de secção transversa (Dalston, 2004, Genaro et al., 2004; Warren, 2004). A nasometria foi utilizada, no presente estudo, para investigar as repercussões acústicas de uma eventual DVF associada à FPSM e a técnica fluxo-pressão, as repercussões aerodinâmicas.

\section{Método}

\section{Casuística}

O estudo foi desenvolvido no Laboratório de Fisiologia do HRAC/USP, com a aprovação do Comitê de Ética em Pesquisa (protocolo no 103/96CEP-UEP), sendo os indivíduos devidamente esclarecidos sobre o estudo e solicitados a assinar o termo de consentimento livre e esclarecido.

Foram avaliados indivíduos convocados entre pacientes com FPSM cadastrados no HRAC, da região de Bauru, ou, identificados entre os casos novos, com base nos seguintes critérios:

\section{Critérios de inclusão}

1. Fissura de palato submucosa isolada ou associada à fissura de lábio.

2. Presença de, pelo menos, dois dos sinais da tríade clássica da FPSM (úvula bífida, diástase muscular e chanfradura óssea).

3. Ausência de sintomas de disfunção velofaríngea, ou seja, com função velofaríngea adequada, segundo avaliação perceptivo-auditiva da fala realizada por fonoaudióloga com experiência na área, conforme detalhado no item seguinte.

4. Idade superior à quatro anos.

\section{Critérios de exclusão}

1. Fissura de lábio não reparada.

2. Síndromes óbvias.

3. Cirurgia de palato prévia.

4. Sintomas respiratórios ou alérgicos que resultassem em congestão nasal ao exame.

5. Incapacidade física e mental para realizar os exames.

Com base nestes critérios, compuseram a amostra um total de 20 pacientes (11 do sexo feminino e 9 do sexo masculino), sendo 8 com FPSM isolada e 12 com FPSM associada à fissura de lábio previamente reparada, com idade entre 6 e 46 anos. Todos apresentavam a tríade clássica de sinais clínicos da FPSM. Uma vez confirmada a ausência de sintomas de DVF na avaliação perceptiva da fala, os pacientes foram submetidos à avaliação acústica e à avaliação aerodinâmica da função velofaríngea.

\section{Avaliação perceptiva da função velofaríngea}

Os pacientes foram submetidos à avaliação perceptiva da fala utilizada de rotina no Laboratório de Fisiologia do HRAC/USP (Trindade e Trindade Junior, 1996). Para a avaliação da função velofaríngea foi adotado o sistema de classificação proposto por Trindade et al., (2005), que se baseia na atribuição de escores a nasalidade, emissão nasal de ar e articulações compensatórias. A hipernasalidade é classificada utilizando uma escala de 6 pontos, sendo $1=$ ausente, $2=$ leve, $3=$ leve para moderada, $4=$ moderada, $5=$ moderada para grave e $6=$ grave. São considerados significativos escores iguais ou superiores a 2. A emissão de ar nasal é classificada de acordo com a quantidade de escape aéreo nasal detectada no teste do espelho, durante o sopro, sopro com a língua protruída, emissão prolongada dos fonemas /i/, /u/, /f/, /s/ e /S/ e de vocábulos e frases com os fonemas plosivos e fricativos, considerando-se uma escala de 6 pontos, sendo $1=$ ausente, $2=$ leve, $3=$ leve para moderado, $4=$ moderado, $5=$ moderado para intenso e $6=$ intenso. São considerados clinicamente significativos escores iguais ou superiores a 3 . A presença de articulações compensatórias é analisada durante a emissão de fonemas plosivos, fricativos e africados e graduada em escala de 2 pontos, sendo $1=$ ausente e $2=$ presente em um ou mais fonemas. A função velofaríngea é, então, classificada como "adequada", "marginal", ou "inadequada", conforme a combinação de escores atribuídos a cada uma das características da fala acima mencionadas, como mostra a Tabela 1 .

Os dados relativos ao grupo de pacientes incluídos no estudo podem ser observados na Tabela 2. Todos apresentaram ausência de hipernasalidade (escore 1), escape aéreo nasal ausente (escore 1) ou leve (escore 2), ausência de articulações compensatórias (escore1). Com base nesses achados a função velofaríngea foi categorizada como adequada e os pacientes considerados assintomáticos. 
TABELA 1. Classificação adotada para avaliar a função velofaríngea segundo escores de hipernasalidade, escape aéreo nasal e articulações compensatórias (Trindade et al., 2005).

\begin{tabular}{c|cccc}
\hline \multicolumn{4}{c}{ Características da Fala } \\
\hline Hipernasalidade* & $\begin{array}{c}\text { Escape Aéreo } \\
\text { Nasal* }\end{array}$ & $\begin{array}{c}\text { Articulações } \\
\text { Compensatórias \# }\end{array}$ & $\begin{array}{c}\text { Função } \\
\text { Velofaríngea }\end{array}$ \\
\hline 1 & 1 & 1 & adequada \\
1 & 2 & 1 & marginal \\
\hline 1 & 3 & $1-2$ & \\
2 & $1-3$ & $1-2$ & inadequada \\
3 & $1-3$ & $1-2$ & \\
\hline 2 & $4-6$ & $1-2$ & $1-2$ \\
3 & $4-6$ & $1-2$ &
\end{tabular}

* 1 ausente; 2 leve; 3 leve para moderado; 4 moderado; 5 moderado para grave; 6 grave.

\# 1 ausente; 2 presente

TABELA 2. Idade, sexo e escores da avaliação perceptiva da fala dos pacientes com fissura de palato submucosa incluídos no estudo.

\begin{tabular}{|c|c|c|c|c|c|c|}
\hline \multicolumn{7}{|c|}{ Avaliação Perceptiva da Fala } \\
\hline Paciente & Sexo & Idade (Anos) & $\begin{array}{c}\text { Articulação } \\
\text { Compensatória }\end{array}$ & Escape Aéreo Nasal & Hipernasalidade & Função Velofaríngea \\
\hline 1 & $\mathrm{M}$ & 6 & 1 & 2 & 1 & adequada \\
\hline 2 & M & 7 & 1 & 2 & 1 & adequada \\
\hline 3 & M & 7 & 1 & 2 & 1 & adequada \\
\hline 4 & M & 10 & 1 & 2 & 1 & adequada \\
\hline 5 & M & 10 & 1 & 1 & 1 & adequada \\
\hline 6 & M & 12 & 1 & 1 & 1 & adequada \\
\hline 7 & M & 16 & 1 & 1 & 1 & adequada \\
\hline 8 & M & 18 & 1 & 2 & 1 & adequada \\
\hline 9 & M & 22 & 1 & 1 & 1 & adequada \\
\hline 10 & $\mathrm{~F}$ & 6 & 1 & 2 & 1 & adequada \\
\hline 11 & $\mathrm{~F}$ & 7 & 1 & 2 & 1 & adequada \\
\hline 12 & F & 9 & 1 & 1 & 1 & adequada \\
\hline 13 & F & 10 & 1 & 2 & 1 & adequada \\
\hline 14 & F & 12 & 1 & 2 & 1 & adequada \\
\hline 15 & F & 12 & 1 & 1 & 1 & adequada \\
\hline 16 & F & 14 & 1 & 2 & 1 & adequada \\
\hline 17 & F & 18 & 1 & 2 & 1 & adequada \\
\hline 18 & F & 26 & 1 & 2 & 1 & adequada \\
\hline 19 & F & 32 & 1 & 1 & 1 & adequada \\
\hline 20 & F & 46 & 1 & 2 & 1 & adequada \\
\hline
\end{tabular}

1 ausente; 2 leve 
Avaliação acústica da função velofaríngea: nasometria

A função velofaríngea foi inferida a partir de medida da nasalância, o correlato físico da nasalidade, utilizando o nasômetro modelo 6200-2 IBM Kay Elemetrics, software versão 30-02-1.7. O sistema é composto por dois microfones posicionados um de cada lado de uma placa de separação sonora, colocada acima do lábio superior do paciente. $\mathrm{O}$ conjunto é mantido em posição por um capacete. $\mathrm{O}$ microfone superior capta os sinais do componente nasal da fala e o inferior, os sinais do componente oral, os quais são filtrados, digitalizados por módulos eletrônicos e processados no microcomputador por meio de um software específico. A nasalância (expressa em \%), corresponde à quantidade relativa de energia acústica nasal na fala, ou seja, à razão numérica entre a quantidade de energia acústica nasal e energia acústica total (soma da energia acústica nasal e oral), multiplicada por 100.

O exame foi realizado durante a leitura de um texto formado por 5 frases do Português Brasileiro, contendo sons exclusivamente orais: Papai caiu da escada. Fábio pegou o gelo. O palhaço chutou a bola. Teresa fez pastel. A árvore dá frutos e flores (Trindade et al., 1997). Para fins de análise, foi considerado o registro da primeira emissão tecnicamente aceitável do texto, ou seja, produzida sem erros e dentro do limite de intensidade aceitável do instrumento.

Avaliação aerodinâmica da função velofaríngea: técnica fluxo-pressão

A função velofaríngea foi também estimada pela medida da área do orifício velofaríngeo durante a fala, por meio da técnica fluxo-pressão, utilizando o sistema computadorizado PERCI-SARS versão 3.0 (Microtronics Corporation). A técnica baseiase no princípio de que a área de secção transversa mínima de uma constrição (orifício velofaríngeo) pode ser estimada pela medida simultânea da diferença de pressão existente entre os dois lados da constrição (pressão oral e pressão nasal) e do fluxo aéreo que a atravessa (fluxo nasal). A área do orifício velofaríngeo é calculada no momento em que a pressão oral alcança o seu valor máximo durante a produção de uma consoante plosiva. As pressões oral e nasal são captadas por catéteres de polietileno conectados a transdutores diferenciais de pressão. Para o registro da pressão oral, um catéter é posicionado na boca e, para o registro da pressão nasal, um segundo catéter é inserido numa rolha, a qual é ajustada na narina de menor fluxo aéreo, criando-se uma coluna de ar estática. O fluxo aéreo nasal é recolhido por um tubo plástico posicionado na narina de maior fluxo, o qual é conectado a um pnemotacógrafo aquecido ligado a um transdutor diferencial de pressão. Os sinais dos três transdutores são processados por um pré-amplificador, enviados ao microcomputador e, analisados por software específico.

A área de secção transversa do orifício velofaríngeo foi calculada durante a produção repetida do fonema /p/ inserido na palavra "rampa", utilizando a equação: $\mathrm{A}=\dot{\mathrm{V}} / \mathrm{k}[2 \mathrm{DP} / \mathrm{d}]^{1 / 2}$, onde $\mathrm{A}=$ área velofaríngea, em $\mathrm{cm}^{2} ; \dot{\mathrm{V}}=$ fluxo aéreo nasal, $\mathrm{em} \mathrm{ml} / \mathrm{s} ; \mathrm{k}=0.65 ; \mathrm{DP}=$ diferença entre a pressão oral e a pressão nasal, em dinas $/ \mathrm{cm}^{2} ; \mathrm{d}=0.001 \mathrm{~g} /$ $\mathrm{cm}^{3}$ (densidade do ar). Para fins de análise, foram considerados os valores médios de quatro a seis produções.

Análise dos dados

Os valores de nasalância foram comparados com os valores normais estabelecidos em estudo desenvolvido no Laboratório de Fisiologia do HRAC/USP em falantes do Português Brasileiro (Trindade et al., 1997).

Teoricamente, o valor da nasalância pode variar de $0 \%$, que corresponde à ausência de som nasal na fala, até $100 \%$, quando todo som emerge do nariz. O escore $27 \%$ foi considerado como o limite superior de normalidade para o texto utilizado. Em outras palavras, valores de nasalância superiores a $27 \%$ foram julgados como indicativos de excessiva quantidade de energia acústica nasal na fala, ou seja, hipernasalidade, resultante de função velofaríngea inadequada. Esse escore corresponde ao valor que maximizou a sensibilidade e especificidade da nasometria em identificar a presença ou ausência de hipernasalidade em outro estudo realizado no Laboratório de Fisiologia do HRAC/USP (Trindade et al., 2003).

$\mathrm{Na}$ avaliação aerodinâmica, a função velofaríngea foi classificada segundo critério proposto por Warren (2004): áreas de 0 a 4,9 $\mathrm{mm}^{2}=$ fechamento adequado, 5 a $9,9 \mathrm{~mm}^{2}=$ fechamento adequado para marginal, 10 a $19,9 \mathrm{~mm}^{2}=$ fechamento marginal para inadequado e, $20 \mathrm{~mm}^{2}$ ou maior $=$ fechamento inadequado.

A hipótese testada no estudo foi a de que, corroborando a avaliação perceptiva da fala (indicativa de função velofaríngea adequada em todos os pacientes incluídos no estudo), as avaliações instrumentais (nasometria e técnica fluxo- 
pressão) seriam também sugestivas de função velofaríngea adequada, ou seja, em todos os pacientes seriam observados valores de nasalância menores ou iguais a 27\% (indicando ausência de hipernasalidade) e valores da área velofaríngea de, no máximo, $4,9 \mathrm{~mm}^{2}$ (indicando fechamento velofaríngeo adequado), respectivamente.

\section{Resultados}

Como já mencionado, todos os indivíduos incluídos no estudo apresentavam função velofaríngea adequada segundo a avaliação perceptiva realizada (Tabela 2 ), caracterizada por ausência de hipernasalidade $(n=20)$, ausência de escape aéreo nasal $(n=7)$ ou escape leve $(n=13)$ e ausência de articulações compensatórias $(\mathrm{n}=20)$, sendo por esta razão considerada assintomáticos.

Com relação à avaliação nasométrica, a Tabela 3 mostra que, no grupo de pacientes estudados, o valor médio de nasalância foi de $20 \pm 10 \%$, sendo que a nasalância mínima observada foi igual a $7 \%$ e a nasalância máxima igual a 46\%. Valores de nasalância normais (ou seja, nasalância menor ou igual a 27\%) foram observados em apenas 15 dos 20 pacientes $(75 \%$ dos casos). Nos outros cinco pacientes (15\% dos casos), identificados na Tabela 3 com um asterisco, os valores de nasalância foram maiores que $27 \%$, sugerindo a presença de hipernasalidade. Na mesma Tabela, verifica-se que em três desses pacientes foram observados valores de nasalância muito próximos ao limite de normalidade (nasalância igual a $28 \%$ nos pacientes 4 e 9 e $30 \%$ no paciente 11 ). Nos outros dois pacientes, entretanto, a nasalância foi bem superior ao limite de normalidade (39\% no paciente 5 e $46 \%$ no paciente 12$)$. Pelo fato desses cinco (15\%) pacientes terem sido diagnosticados como sintomáticos pela nasometria, e, como assintomáticos na avaliação perceptiva, foi, neste caso, descartada a hipótese de nulidade em análise, não cabendo nenhum tratamento estatístico adicional dos dados.

Com relação à análise aerodinâmica, o grupo apresentou, em média, uma área velofaríngea de $0,9 \pm 1,3 \mathrm{~mm}^{2}$, sendo que a área mínima observada foi de $0 \mathrm{~mm}^{2}$ e a máxima foi de $4,8 \mathrm{~mm}^{2}$. De acordo com escala de classificação de Warren (2004), esses valores são todos indicativos de fechamento velofaríngeo adequado. Constatou-se portanto, que os pacientes foram categorizados como assintomáticos tanto na avaliação aerodinâmica como na avaliação perceptiva.

A comparação do desempenho das três modalidades de avaliação da função velofaríngea utilizadas no presente estudo, perceptiva, nasométrica e aerodinâmica, é resumida na Tabela 4.

TABELA 3. Valores individuais e médios de nasalância, expressos em porcentagem, e da área de secção transversa do orifício velofaríngeo, expressa em $\mathrm{mm}^{2}$, obtidos na avaliação nasométrica e aerodinâmica, respectivamente.

\begin{tabular}{|c|c|c|}
\hline Paciente & Nasalância (\%) & Área Velofaríngea (mm) \\
\hline 1 & 8 & 0,1 \\
\hline 2 & 8 & 0,4 \\
\hline 3 & 18 & 1,5 \\
\hline 4 & $28^{*}$ & 0,7 \\
\hline 5 & $39^{*}$ & 0,8 \\
\hline 6 & 24 & 0,4 \\
\hline 7 & 20 & 0,2 \\
\hline 8 & 22 & 0,2 \\
\hline 9 & $28^{*}$ & 4,8 \\
\hline 10 & 21 & 2,9 \\
\hline 11 & $30^{*}$ & 3,0 \\
\hline 12 & $46^{*}$ & 0,1 \\
\hline 13 & 17 & 0,1 \\
\hline 14 & 16 & 0,1 \\
\hline 15 & 7 & 0 \\
\hline 16 & 11 & 0,9 \\
\hline 17 & 15 & 0,6 \\
\hline 18 & 25 & 0,3 \\
\hline 19 & 9 & 0,1 \\
\hline 20 & 16 & 0,2 \\
\hline valor mínimo & 7 & 0 \\
\hline valor máximo & 46 & 4,8 \\
\hline $\begin{array}{l}\text { média } \pm \text { desvio } \\
\text { padrão }\end{array}$ & $20 \pm 10$ & $0,9 \pm 1,3$ \\
\hline
\end{tabular}

*valores anormais sugestivos de hipernasalidade (nasalância maior que 27\%)

TABELA 4. Número (porcentagem) de pacientes com diagnóstico de função velofaríngea adequada e inadequada na avaliação perceptiva e nas avaliações instrumentais (nasometria e técnica fluxo-pressão).

\begin{tabular}{c|c|c}
\hline & Função Velofaríngea & Função Velofaríngea \\
Modalidade de Avaliação & Adequada & Inadequada \\
\hline avaliação perceptiva & $20(100 \%)$ & $0(0 \%)$ \\
nasometria & $15(75 \%)^{*}$ & $5(15 \%)^{*}$ \\
técnica fluxo-pressão & $20(100 \%)$ & $0(0 \%)$ \\
\hline
\end{tabular}

*hipótese de nulidade descartada (ausência de concordância total entre nasometria e avaliação perceptiva) 


\section{Discussão}

Para o desenvolvimento do presente trabalho, a questão que se colocou foi se pacientes com FPSM não estariam sendo indevidamente considerados assintomáticos a partir de uma análise perceptiva da fala e dispensados do atendimento devido. Diante dos resultados encontrados em 20 pacientes submetidos a duas diferentes modalidades de avaliação instrumental, podemos inferir que a resposta é positiva para cinco dos casos analisados. Confirmando hipótese inicial, houve total concordância entre os achados da avaliação perceptiva e a avaliação aerodinâmica. Entretanto, em alguns casos, foi observada discrepância entre a avaliação perceptiva e a nasometria, o que pode ter importante significado clínico.

No grupo de 20 pacientes incluídos no estudo, por terem sido considerados assintomáticos na avaliação perceptiva, valores de nasalância anormais foram observados em cinco pacientes, o que corresponde a $15 \%$ do total de casos analisados. Foi descartada, desta forma, a hipótese de que todos exibiriam valores normais de nasalância. Isto pode significar que nesses cinco pacientes houve falha no julgamento perceptivo da nasalidade ou que a nasometria detectou desvios de ressonância sutis não perceptíveis ao ouvido humano. Como mostrado na Tabela 3 , há que se considerar que três desses pacientes apresentaram valores de nasalância limítrofes, ou seja, muito próximos ao limite superior de normalidade de $27 \%$ estabelecido em estudo desenvolvido no Laboratório de Fisiologia do HRAC/USP (Trindade et al., 2003). Não se pode, igualmente, excluir a possibilidade de erro nas medidas feitas com a nasometria, inclusive por contaminação decorrente de transmissão acústica transpalatina (Gildersleeve-Neumann e Dalston, 2001), passível de ocorrer especialmente nos casos de fissura de palato submucosa. Nos outros dois pacientes, entretanto, a nasalância foi nitidamente superior ao limite de normalidade, sugerindo de maneira mais contundente a presença de hipernasalidade. É importante destacar que os valores de nasalância observados nesses dois casos (39\% e $46 \%$ ) foram próximos ao encontrado, em média, em fissurados de palato portadores de DVF (42 $\pm 8 \%$ ), em outro estudo do Laboratório de Fisiologia do HRAC/USP conduzido por Zuiani et al., (1998). A identificação de evidências de DVF em cinco (ou mesmo em dois) pacientes pela nasometria, não detectada na avaliação perceptiva, pode significar a necessidade de mudança na conduta de tratamento oferecido a esses pacientes.

Quanto às medidas das dimensões da área velofaríngea, estas se situaram, em todos os casos, abaixo do limite de normalidade de $5 \mathrm{~mm}^{2}$ (Warren, 2004) confirmando, portanto, as impressões da avaliação perceptiva de que estes pacientes apresentavam função velofaríngea adequada. A concordância entre as avaliações aerodinâmica e perceptiva, em si, parece invalidar os resultados da avaliação nasométrica. No entanto, dois fatos falam a favor das observações aferidas na nasometria: primeiro, na avaliação aerodinâmica, a função velofaríngea é inferida a partir da área do orifício velofaríngeo medida na produção de um único som consonantal, enquanto que a medida da nasalância é feita durante a produção de uma amostra de fala que contém diferentes sons vocálicos e consonantais, mais próxima, portanto, à fala espontânea. Segundo, nos cinco pacientes com nasalância aumentada poderia estar ocorrendo o problema de "timing" no funcionamento do esfíncter velofaríngeo (Dotevall et al., 2002; Zajac e Hackett, 2002; Warren, 2004), levando à hipernasalidade na presença de fechamento velofaríngeo adequado, o que explicaria as discrepâncias observadas entre os dois métodos.

A identificação de evidências de hipernasalidade com a nasometria, em, pelo menos dois dos pacientes com FPSM estudados, reforça a importância do uso combinado de métodos perceptivos e instrumentais e, mais do que isto, fortemente recomenda o acompanhamento, criterioso e periódico, dos casos nos quais a avaliação instrumental aponta para sinais de disfunção do mecanismo velofaríngeo, contrariando as impressões clínicas. Isto é particularmente verdadeiro para crianças que ainda não têm estruturas e mecanismos do trato vocal completamente desenvolvido, estando, por esta razão, mais sujeitas a desenvolver os sintomas próprios da DVF com o avanço da idade. Idealmente, esses sintomas devem ser identificados o mais precocemente possível para que sejam adotadas condutas terapêutico-cirúrgicas mais adequadas, que levem a resultados mais favoráveis, impedindo o desenvolvimento dos distúrbios de fala. Considerando que a nasometria e a técnica fluxo-pressão têm aplicação limitada a crianças de maior idade, há que se desenvolver métodos instrumentais que permitam a avaliação dos sintomas da DVF já na primeira infância, de maneira similar ao que se fez no presente estudo. 


\section{Referências Bibliográficas}

BATTAIOLA, C. M.; YAMAShitA, R. P. Revisão cirúrgica do retalho faríngeo. Salusvita, Bauru, v. 24, n. 1, p. $75-85,2005$.

DALSTON, R. M. The use of nasometry in the assessment and remediation of velopharyngeal inadequacy. In: $\mathrm{BZOCH}, \mathrm{K}$. R. (Ed.). Communicative disorders related to cleft lip and palate. Austin: Pro-ed, 2004. p. 493-516.

DOTEVALL, H.; LOHMANDER-AGERSKOV, A.; EJNELL, H.; BJÖRN, B. Perceptual evaluation of speech and velopharyngeal function in children with and without cleft palate and relationship to nasal airflow patterns. Cleft Palate Craniofac. J., Chapel Hill, v. 39, n. 4, p. 409424, jul. 2002.

GENARO, K. F.; YAMASHITA, R. P.; TRINDADE, I. E. K. Avaliação clínica e instrumental na fissura labiopalatina. In: FERREIRA, L. P.; BEFI-LOPES, D. M.; LIMONGI, S. C. O. (Org.). Tratado de fonoaudiologia. São Paulo: Roca, 2004. p. 456-477.

GILDERSLEEVE-NEUMANN, C. E.; DALSTON, R. M. Nasalance scores in noncleft individuals: why not zero? Cleft Palate Craniofac. J., Chapel Hill, v. 38, n. 2, p. 106111, mar. 2001.

JOHNS, D. F.; ROHRICH, R. J.; AWADA, M. Velopharyngeal incompetence: a guide for clinical evaluation. Plast. Reconstr. Surg., Hagerstown, v. 112, n. 7, p. 1890-1897, dec. 2003.

KUMMER, A. W. Velopharyngeal dysfunction (VPD) and resonance disorders. In: KUMMER, A. W. Cleft palate \& craniofacial anommalies: effects of speech and resonance. San Diego: Singular, 2001. cap. 7, p. 145-176.

MCWILLIAMS, B. J. Submucous clefts of the palate: how likely are they to be symptomatic. Cleft Palate Craniofac. J., Chapel Hill, v. 28, n. 3, p. 247-251, jul. 1991.

OLIVEIRA, R. P. Relação entre os sinais clínicos da fissura de palato submucosa e a sintomatologia específica: uma abordagem preventiva. 2002. 103 f. Dissertação (Mestrado em Distúrbios da Comunicação Humana) Hospital de Reabilitação de Anomalias Craniofaciais, Universidade de São Paulo, Bauru.

PARK, S. Cephalometric analysis insubmucous cleft palate: comparison of cephalometric data obtained form submucous cleft palate patients with velpharyngeal competence and incompetence. Cleft Palate Craniofac. J., Chapel Hill, v. 39, n. 1, p. 105-109, jan. 2002.

PARK, S.; SASSO, Y.; ITO, O.; TOKIAKA, K.; KATO, K.; NITTA, N.; KITANO, I. A Retrospective study of speech development in patients with submucous cleft palate treated by four operations. Scand. J. Plast. Reconstr. Surg. Hand Surg., Hagerstone, v. 34, n. 2, p. 131-136, jun. 2000.

SENDERS, C. W. Management of velopharyngeal competence. Facial Plast. Surg. Clin. North Am., Philadelphia, v. 9, n. 1, p. 27-35, feb. 2001.
SOMMERLAD, B. C.; MEHENDALE, F. V.; BIRCH, M. J.; SELL, D.; HATTEE, C.; HARLAND, K. Palate rerepaired revisited. Cleft Palate Craniofac. J., Chapel Hill, v. 39, n. 3, p. 295-307, may 2002.

SOMMERLAD, B. C.; FENN, C.; HARLAND, K.; SELL, D.; BIRCH, M. J.; DAVE, R.; LEES, M.; BARNETT, A. Submucous cleft palate: a grading system and review of 40 consecutive submucous cleft palate repairs. Cleft Palate Craniofac. J., Chapel Hill, v. 41, n. 2, p. 114-123, mar. 2004.

TRINDADE, I. E. K.; TRINDADE JUNIOR, A. S. Avaliação funcional da inadequação velofaríngea. In: CARREIRÃO, S.; LESSA, S.; ZANINI, S. A. (Ed.). Tratamento das fissuras labiopalatinas. 2. ed. Rio de Janeiro: Revinter, 1996. cap. 26, p. 223-235.

TRINDADE, I. E. K.; GENARO, K. F.; DALSTON, R. M. Nasalance scores of normal Brazilian Portuguese speakers. Braz. J. Dysmorphol. Speech Hear. Disord., Bauru, v. 1, n. 1, p. 23-34, jul. 1997.

TRINDADE, I. E. K; YAMASHITA, R. P.; SUGUIMOTO, R. M.; MAZZOTTINI, R.; TRINDADE JUNIOR, A. S. Effects of orthognathic surgery on speech and breathing of subjects with cleft lip and palate: acoustic and aerodynamic assessment. Cleft Palate Craniofac. J., Chapel Hill, v. 40, n. 1, p. 54-64, jan. 2003.

TRINDADE, I. E. K; GENARO, K. F.; YAMASHITA, R. P.; MIGUEL, H. C.; FUKUSHIRO, A. P. Proposta de classificação da função velofaríngea na avaliação perceptivo-auditiva da fala. Pró-Fono R. Atual. Cient., Barueri (SP), v. 17, n. 2, p. 259-262, maio-ago. 2005.

WARREN, D. W. Aerodynamic assessment and procedures to determine extend of velopharyngeal inadequacy. In: $\mathrm{BZOCH}, \mathrm{K}$. R. (Ed.). Communicative disorders related to cleft lip and palate. Austin: Pro-ed, 2004. p. 595-628.

YAMASHITA, R. P.; CALAIS, L. L.; MIGUEL, H. C.; TRINDADE, I. E. K. Avaliação da resistência laríngea em indivíduos portadores de insuficiência velofaríngea com distúrbio articulatório compensatório. Acta Awho, São Paulo, v. 21, n. 2, abr.-jun. 2002. Disponível em: <htpp:/ /www.actaawho.com.br/>. Acesso em: 20 set. 2006.

YSUNZA, A.; PAMPLONA, M. C.; MENDONZA, M.; MOLINA, F.; MARTINEZ, P.; GARCIA-VELASCO, M.; PRADA, N. Surgical treatment of submucous cleft palate: a comparative trial of two modalities for palatal closure. Plast. Reconstr. Surg., Hagerstone, v. 107, n. 1, p. 9-14, jan. 2001.

ZAJAC, D. J.; HACKETT, A. M. Temporal characteristics of aerodynamic segments in the speech of children and adults. Cleft Palate Craniofac. J., Chapel Hill, v. 39, n. 4, p. 432-438, jul. 2002.

ZUIANI, T. B. B.; TRINDADE, I. E. K.; YAMASHITA, R. P.; TRINDADE JR, A. S. The pharyngeal flap surgery in patients with velopharyngeal insufficiency: perceptual and nasometric speech assessment. Braz. J. Dysmorphol. Speech. Dis., Bauru, v. 2, n. 1, p. 31-42, jul. 1998. 